\title{
Introducción de Taxones Domésticos y Control de Camélidos en el Norte Semiárido: Variabilidad Osteométrica en el Valle de Mauro, IV Región, Chile
}

\author{
Control and Introduction of Camelid Domestic Taxa in Semiarid North: Osteometric \\ Variability in Mauro Valley, IV Región, Chile
}

Isabel Cartajena Fasting, Bárbara Rivera ${ }^{\text {i }}$, Patricio López ${ }^{\text {ii }}$ y Boris Santanderiii

\begin{abstract}
RESUMEN
En el presente trabajo se dan a conocer los resultados del análisis osteométrico de los sitios provenientes del Valle de Mauro (MAU033, MAU085, MAU062, MAU065, MAU083, MAU09I, MAU064, MAU092, MAU067 y MAU094), Norte Semiárido, Chile. Los análisis cubren una secuencia temporal que abarca desde el Arcaico Tardío hasta el Período Tardío. En este trabajo se busca evaluar la hipótesis que señala la irrupción de taxones domésticos (llama) tardíamente durante el periodo Diaguita-Inka. Los resultados de los sitios arcaicos presentaban en algunos casos un tamaño mayor que los estándares actuales de guanaco utilizados como referencia. Dada la adscripción cronológica de los yacimientos, los restos se interpretaron como guanacos pero con un morfotipo más grande que los utilizados como referencia. En el caso de los sitios tardíos se concluye que los restos representados corresponderían al menos a dos taxones diferentes del grupo de camélidos grandes, guanaco y llama. No obstante, al observar los resultados diacrónicamente se observa una alta variabilidad osteométrica que dificulta las interpretaciones en cuanto a los taxones presentes y sugieren un posible control temprano de los camélidos.
\end{abstract}

Palabras Clave: Camélidos Sudamericanos, Osteometría, Norte Semiárido, Periodo Arcaico Tardío, Intermedio Tardío, Tardío

i Departamento de Antropología, Universidad de Chile, Ignacio Carrera Pinto 1045, Nuñoa, Santiago. Correo-e: icartaje@uchile.cl, barbirivera@gmail.com.

ii Programa de Doctorado en Antropología, Universidad Católica del Norte, IIAM. Gustavo Le Paige 380, San Pedro de Atacama. Correo-e: patricio.lopezmendoza@yahoo.es

iii Programa de Doctorado en Cuaternario y Prehistoria, Universitat Rovira i Virgili. Institut Català de Paleoecología Humana i Evolució Social (IPHES), Catalunya, España. Grupo "Quaternário e Pré-História" do Centro de Geociências, uID73 - FCT, Portugal. Correo-e: boris.santander@gmail. com

Recibido: 01-02-2013 Revisado: 15-01-2014 Aceptado: 21-03-2014 


\section{ABSTRACT}

Osteometric analysis results of the bone assemblages from the sites located in Valle de Mauro (MAU033, MAU085, MAU062, MAU065, MAU083, MAU09I, MAU064, MAU092, MAU067 and MAU094), Semiarid North, Chile are presented. The sites cover an occupational sequence from the Late Archaic to the Late Period. The late introduction of domestic taxa (llama) during the Diaguita-Inka period is re-evaluated. Results show the presence of big sized camelids, even bigger in size than modern guanacos used as reference during the Late archaic. For the later period two taxa, llama and guanaco, are represented.Although, high osteometric variability along the sequence difficults a more accurate interpretation, an early control of domestic camelids is suggested.

Key Words: Southamerican Camelids, Osteometry, Semiarid North, Late Archaic, Late Intermediate, Late Period

\section{INTRODUCCIÓN}

En general ha prevalecido una interpretación histórico cultural para los grupos que habitaron el Norte Semiárido, en la cual se asocian los primeros grupos ceramistas con un modelo agroganadero en el cual se incorporan tempraneramente los camélidos domésticos (Gallardo 1997). Si bien hallazgos como los entierros de Plaza Coquimbo denotarían la importancia de los camélidos domésticos para los grupos humanos (Castillo 1984), nuevos trabajos señalarían que la incorporación de la llama es relativamente tardía (Diaguita) y que iría de la mano con la influencia del Tawantinsuyu (Troncoso et al. 2009).

No obstante, los trabajos referidos a los restos arqueofaunísticos en esta región son escasos. El estudio de los conjuntos provenientes de Plaza Coquimbo y de otros sitios de filiación Diaguita, no arrojaron resultados concluyentes acerca de la asignación taxonómica de los camélidos representados. Por una parte se observaron diferencias en cuanto al tamaño de las poblaciones silvestres costeras e interiores, donde las últimas presentaban un tamaño mayor. Por otra, al comparar las medidas con sitios diaguitas, los restos de Plaza Coquimbo presentaban en general un tamaño mas homogéneo pero más pequeño (Becker 2005, 2006).

Los sitios analizados en este trabajo provienen del Valle de Mauro (Comuna de Los Vilos) $31^{\circ} 57^{\prime} \mathrm{S}-71^{\circ} 0 I^{\prime} \mathrm{O}$, ca. 900 m.s.n.m. y representan una secuencia ocupacional desde el Arcaico hasta el Periodo Tardío (ver Rivera et al., Figura I en este volumen). El material óseo recuperado en la excavación de los diversos sitios es abundante, lo que permitió realizar comparaciones entre los diversos yacimientos. Se puso especial hincapié en la identificación de posibles cambios en la composición taxonómica referida al conjunto de los camélidos.

Los yacimientos arcaicos corresponderían a campamentos abiertos de cazadores-recolectores especializados en la caza de guanacos insertos en un circuito de movimientos estacionales $(\sim 3.000$ -2.300 A.P.). De igual forma, los primeros grupos portadores de cerámica también habrían dependido fuertemente de la caza y recolección manteniendo una fuerte movilidad (Méndez y Jackson 2008). El Período Intermedio Tardío ( 1.000-1.400 años d.C.) se encuentra caracterizado principalmente en esta region por la cutura Diaguita, una sociedad de base agrícola con una explotación a baja escala del entorno a pesar que se reconoce el contacto con ambientes más lejanos como la costa (Troncoso 1999 , 200I). Entre los productos explotados se encuentran cultivos de alto rendimiento como la quínoa y el maíz (Belmar y Quiroz 2004) y además, el guanaco adquirido a través de la caza. Hacia el Periodo Tardío ( 1400-1450), se incorporaría además la llama, junto a una mayor diversificación de los recursos a la llegada del Tawantinsuyu (Becker 2004, Troncoso 2004).

\section{MATERIAL Y MÉTODO}

Para la determinación taxonómica de los restos de camélidos se utilizaron técnicas osteométricas a partir de un conjunto de 279 especimenes medidos (Tabla I) para lo que se consideraron primeras y segundas falanges anteriores como posteriores y astrágalos enteros o bien, en aquellos casos en que se encontraban fracturados, al menos una porción lo suficientemente completa para la obtención de alguna de sus medidas, privilegiando los restos de animales adultos que hayan alcanzado su tamaño definitivo (von den Driesch 1999). 


\begin{tabular}{|l|c|c|c|c|c|c|c|}
\hline \multicolumn{1}{|c|}{ Sitio } & Periodo & $\begin{array}{c}\text { I Falange } \\
\text { anterior }\end{array}$ & $\begin{array}{c}\text { I Falange } \\
\text { posterior }\end{array}$ & $\begin{array}{c}\text { II Falange } \\
\text { anterior }\end{array}$ & $\begin{array}{c}\text { II Falange } \\
\text { posterior }\end{array}$ & Astrágalo & Total \\
\hline MAU 33 & AT & I & 8 & - & - & 2 & 3 \\
\hline MAU 85 & AT/PAT & 9 & - & I & I & 7 & 26 \\
\hline MAU 62 & PAT & I & - & - & - & - & I \\
\hline MAU 65 & PIT & I & - & - & - & - & I \\
\hline MAU 83 & PIT & 2 & - & - & I & 2 & 5 \\
\hline MAU 9I & PIT & I & I & 2 & 5 & - & 9 \\
\hline MAU 92 & PIT & - & - & - & - & 2 & 2 \\
\hline MAU 64 & PIT/PT & - & I & - & - & 2 & 3 \\
\hline MAU 67 & PIT/PT & 24 & 3 & 8 & 19 & 5 & 59 \\
\hline MAU 94 & PIT/PT & 57 & 31 & 31 & 50 & $\mathbf{2}$ & 170 \\
\hline Total & & $\mathbf{9 6}$ & $\mathbf{4 4}$ & $\mathbf{4 2}$ & $\mathbf{7 6}$ & $\mathbf{2 1}$ & $\mathbf{2 7 9}$ \\
\hline
\end{tabular}

Tabla 1: Primeras falanges, segundas falanges y astrágalos medidos en cada uno de los sitios correspondientes a los periodos Arcaico Tardío (AT), Alfarero Temprano (PAT), Intermedio Tardío (PIT) e Intermedio Tardío-Tardío (PIT/PT).

Table 1: Meassured first phalanxes, second phalanxes and astragali of the sites belonging to the Late Archaic (AT), Early Ceramic Period (PAT), Late Intermediate (PIT) and Late intermediate-Late (PIT/PT) periods.

Se utilizaron gráficos de dispersión con el fin de visualizar en un plano cartesiano las distribuciones de pares de medidas provenientes de los especimenes con fechados taxón, escogiéndose aquel par de variables que discriminó mejor entre los grupos. Las medidas obtenidas se compararon con aquellas provenientes de esqueletos de referencia actuales pertenecientes a Lama guanicoe (guanaco macho adulto) de la Zona Central y una llama adulta proveniente del norte del país, ambos depositados en el Departamento de Antropología de la Universidad de Chile.

Se utilizó además el Índice de Tamaño Logarítmico (LSI) que permitió maximizar el número de medidas, ya que consideraron diversas partes del esqueleto (primeras, segundas falanges y astrágalos) en conjunto (Meadow 1999). Como estándar se utilizó un guanaco macho adulto proveniente de la zona cordillerana de Chile Central. A través de los gráficos obtenidos fue posible comparar las tendencias observables para cada uno de los grupos a través del tiempo con el fin de identificar cambios en el tamaño de los animales que pudiesen dar cuenta de diferencias de tamaño relacionadas con la composición taxonómica.

\section{RESULTADOS DEL ANÁLISIS OSTEOMÉTRICO}

Los especímenes representados durante el Arcaico Tardío superan en talla tanto a los guanacos actuales del Centro de Chile como a las llamas provenientes del Norte Grande. Sin embargo, dada la adscripción cronológica de los sitios (Arcaico Tardío), los restos se interpretaron como guanacos pero con un morfotipo más grande que los utilizados como referencia. Esta tendencia se puede observar claramente en el caso del astrágalo, en el cual los especimenes arqueológicos medidos de ambos sitios (MAU033 y MAU085) presentan un tamaño muy similar, mayor al guanaco utilizado como referencia (Figura I). No obstante, también se encuentran algunas en el rango de los guanacos modernos, lo que sugiere animales de mayor tamaño y variabilidad para los momentos tempranos. 


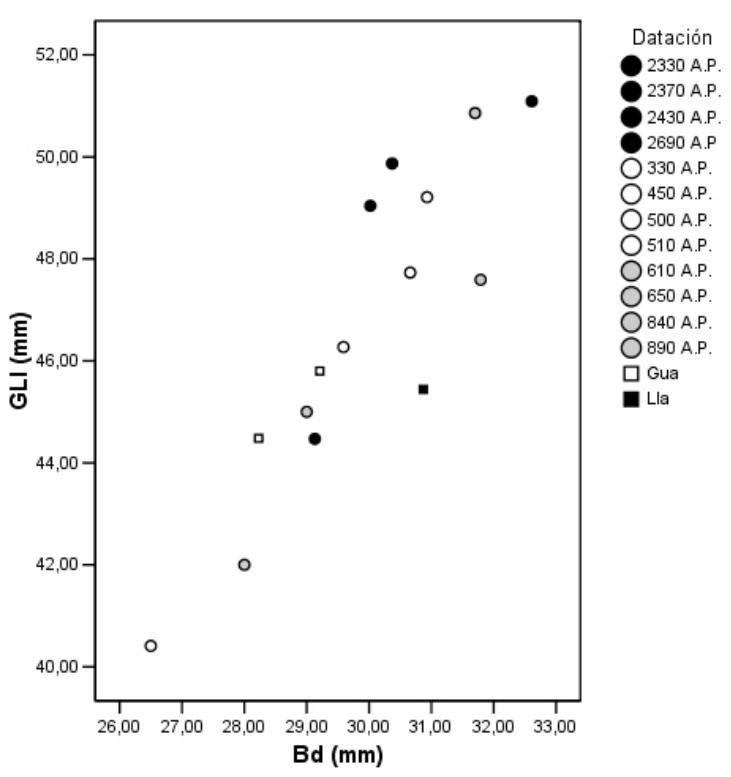

Figura 1: Representación de las medidas largo lateral (GLl) y ancho distal (Bd sensu von den Driesch 1999) para el astrágalo. Las medidas representadas corresponden a fechas taxón obtenidas para los astrágalos de los periodos Arcaico Tardío (2330-2690 A.P.), Periodo Intermedio (890-610 A.P.) y Periodo Tardío (510330 A.P.). Se incluyen medidas de referencia de guanaco (Gua) y llamas (Lla) actuales.

Figure 1: Greatest length lateral (GLl) and distal breadth (Bd sensu von den Driesch 1999) of the astragali. The measuremtes were taken from taxon dated bones from the Late Archaic (2330-2690 A.P.), Late Intermediate (890-610 A.P.) and Late periods (510330 A.P.). also modern guanaco (Gua) and llama (Lla) reference measurements are included.

Por su parte, las medidas obtenidas para el Intermedio Tardío sugieren que los especímenes se mantienen dentro del rango de variación del observado para el Arcaico Tardío, si bien se encuentra un espécimen mucho más pequeño. Al considerar otras medidas inscribibles a sitios del Periodo Intermedio Tardío pero que no tienen fechas taxón, también se encuentran en la parte inferior del rango (Cartajena et al. 2012, Lopez et al. 2012). Esta distribución se observa también para los especímenes medidos del Intermedio Tardío/Tardío. Llama la atención una primera falange posterior que presenta un tamaño muy pequeño en comparación al resto del conjunto ubicándose en el límite inferior del rango de medidas (Figura 2). Las medidas son un poco mayores que las alpacas modernas utilizadas como referencia y otros conjuntos contemporáneos con abundante representación de camélidos pequeños (Cartajena 2002, 2009a). Si bien no encontramos otros especimenes pequeños podría estar evidenciando la presencia de un tercer taxón del grupo de camélidos pequeños (vicuña/alpaca).

Como se mencionó anteriormente para el Índice de Tamaño Logarítmico se consideraron todas las medidas de las primeras, segundas falanges $y$ astrágalos en conjunto (Figura 2). La mayor parte de las medidas provienen de sitios donde se observan componentes del Periodo Intermedio Tardío y Tardío, además las medidas de los sitios monocomponentes del Intermedio Tardío eran escasas por lo que fueron consideradas en conjunto.

Al igual como se observa en la figura anterior, durante el Arcaico Tardío la mediana de los camélidos representados es mayor que el esqueleto de guanaco de la zona Central de Chile utilizado como estándar. Sin embargo, al considerar un mayor numero de medidas la dispersión es menor, denotando una mayor homogeneidad en los especimenes representados. Contrariamente, en el caso del Intermedio Tardío/ Tardío, la mediana decrece y la varianza aumenta, denotando un conjunto mucho más heterogéneo, especialmente en la parte inferior del rango.

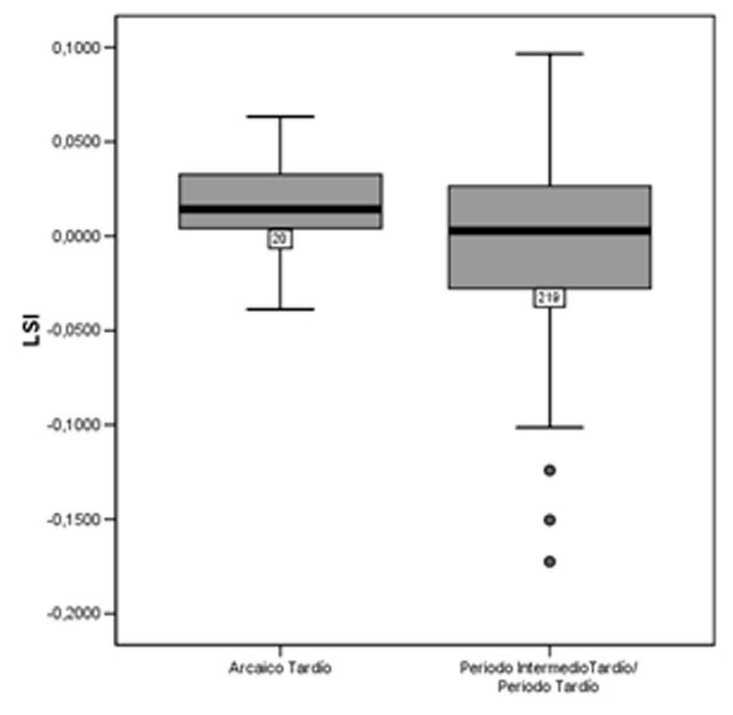

Figura 2: Gráfico de cajas del Indice de Tamaño Logarítmico (LSI) representando medidas obtenidas de las primeras, segundas falanges y astrágalos de los periodos Arcaico Tardío e Intermedio Tardío/ Tardío.

Figure 2: LSI boxplot with meassuremest taken on the first, second phalanxes and astragali of the Late archaic, Late Intermediate/Late pediods. 
Esta tendencia podría explicarse por una parte como un decrecimiento de las especies silvestres, resultante de cambios climáticos que pudieron haber incidido en la disponiblidad y distribucion de los recursos forrajeros. No obstante, los datos paleoclimáticos sugieren a grandes rasgos un periodo de mayor aridez entre los $\sim 3000$ a 2200 A.P., seguido por una fase de mayor humedad a partir de los 2200 A.P. con una alternada de condiciones áridas y húmedas (Maldonado y Villagrán 2002, 2006).

Otra explicación alternativa sería la introducción de taxones domésticos criados localmente, posiblemente desde el Periodo Intermedio Tardío. Sin embargo, esto sería más claro en el Periodo Tardío donde por una parte se observan algunos especímenes de gran tamaño, que superan la talla de los guanacos durante el Arcaico Tardío, lo que ha sido interpretado como llamas con un morfotipo grande, posiblemente cargueras. El decrecimiento de la mediana y el aumento de observaciones en el rango inferior sugieren un conjunto compuesto por una parte por lo que serían llamas con un morfotipo más pequeño, criadas localmente, no pudiéndose descartar la presencia de especies silvestres cazadas, en una zona en que las medidas de guanaco y llama se traslapan.

Llama la atención una primera falange posterior que presenta un tamaño muy pequeño en comparación al resto del conjunto ubicándose en el límite inferior del rango de medidas (Figuras I y 2). Si bien no encontramos otros especimenes pequeños podría estar evidenciando la presencia de un tercer taxón del grupo de camélidos pequeños. No obstante, a diferencia de otros sitios tardíos (Finca Chañaral, III Región) donde la presencia de camélidos pequeños (interpretados como vicuñas) es abundante, en este yacimiento se encuentran escasamente representados. Las medidas tanto de primeras falanges como de segundas son de mayor tamaño que las registradas por ejemplo en Finca Chañaral (Cartajena 2009b).

\section{CONSIDERACIONES FINALES}

Los resultados alcanzados permiten observar el comportamiento de los conjuntos a lo largo de una secuencia ocupacional desde el
Arcaico Tardio. Llama la atencion el gran tamaño de los camélidos arcaicos, lo cual cobra especial relevancia a la hora de interpretar los rangos de tamaños durante los periodos posteriores donde es esperable mayores variaciones en la parte superior del rango con la introducción de taxones domésticos a la llegada del Inka. No obstante, la amplia presencia de especimenes más pequeños que los guanacos arcaicos nos permite postular tentativamente la incorporacion de animales domésticos (llamas), posiblemente desde el Intermedio Tardio. No obstante, se trataría de grupos con un fuerte sustento agrícola, donde la crianza de llamas podría estar mas bien relacionada con el aprovechamiento de fibras textiles más que el aprovisionamiento de carne, el cual está cubierto en parte por la cacería de animales silvestres (guanacos). Sin duda, con la llegada del Tawantinsuyu, la utilización de la llama cobraría especial relevancia, ya sea como animal carguero para articular zonas distantes insertas en una amplia red de circulación o bien como recurso carneo y lanero.

Los resultados, sin duda nos llevan a reflexionar acerca de la naturaleza de las primeras poblaciones alfareras y el caracter pastoralista de éstas. Las variaciones de tamaño detectadas a lo largo de la secuencia, permiten aventurar que la llama podría haber sido introducida recién a partir del Periodo Intermedio Tardio y que con certeza se encuentra presente en los yacimientos del Periodo Tardío.

Agradecimientos: Nuestros agradecimientos a Daniel Pavlovic y Daniel Pascual por su ayuda durante el proceso de análisis. Esta investigación fue financiada por el Proyecto Arqueológico Mauro realizado por el Departamento de Antropología de la Universidad de Chile.

\section{BIBLIOGRAFÍA}

Becker, C. 2004 "Animales que cuentas historias". Chungará 36 (I): 359-364.

2005. "Desempolvando viejas cajas: Estudio de la colección Plaza Coquimbo". Informe Fondo de Investigación Patrimonial DIBAM 25-03-192042.

2006. "Arqueofaunas, investigar y poner el valor las colecciones Diaguitas”. Informe Fondo de Investigación Patrimonial FIP $\mathrm{N}^{\circ}$ 045. Ms

Belmar, C. y L. Quiroz. 2004 "Recursos vegetales y 
modos de explotación: $i Q u e ́$ nos dicen las plantas acerca de sus consumidores? Un estudio de sitios Diaguita en los valles de Illapel y Chalinga". Revista Werken (5): 53-60.

Cartajena, I, P. López, B. Santander y B. Rivera. 2012. Informe de los restos arqueofaunísticos del Valle del Mauro, IV Región, Chile. Manuscrito en poder de los autores.

Cartajena, I. 2002 Los conjuntos arqueofaunísticos del Arcaico Temprano en la Puna de Atacama, Norte de Chile.Tesis doctoral. Freie Universität Berlin. Impresa en microfilm, ABESY Vertriebs $\mathrm{GmbH}$, Alemania [2003].

2009a. "Exploranda la variabilidad morfométrica del conjunto de camélidos pequeños durante el Arcaico Tardío y el Formativo Temprano en quebrada Tulán, norte de Chile". Revista del Museo de Antropología 2: 199-2 12.

2009b. Análisis de los restos arqueofaunísticos del sitio Finca de Chañaral. Informe Qapacñam, no publicado.

Castillo, G. 1984. "Un cementerio del Complejo Las Ánimas en Coquimbo: Ejemplo de relaciones con San Pedro de Atacama”. Estudios Atacameños 7: 199-209 (1984).

Driesch, A. von den. 1999. A guide to measurement of animal bones from archaeological sites. Peabody Museum Bulletins I (5ta. Edición).

Gallardo, F. 1997. "El norte verde y su prehistoria. La tierra donde el desierto florece". En: Chile antes de Chile. Museo Chileno de Arte Precolombino, Santiago, pp. 33-43.

López, P., I. Cartajena, B. Santander, B. Rivera y C. Opazo. 2012. "Explotación de camélidos de un sitio Intermedio Tardío (I.000-I.400 d.C.) y Tardío (I.400-I.536 d.C.) delValle de Mauro (IV Región, Chile)". Boletín de la Sociedad Chilena de Arqueología. En prensa.

Maldonado, A. y C. Villagrán. 2002 "Paleoenvironmental changes in the semiarid coast of Chile $\left(\sim 32^{\circ} \mathrm{S}\right)$ during the last $6200 \mathrm{cal}$ years inferred from a swampforest pollen record". Quaternary Research 58:I30-138.
2006. "Variability of the Northern limit of the Southern Westerlies over the last $9900 \mathrm{cal}$ yr BP from a swamp forest pollen record along the semiarid coast of Chile ( $\left.32^{\circ} 05^{\prime} \mathrm{S}\right)$ ". Quaternary Research 66:246-258.

Meadow, R. H. 1999. "The use of size index scaling techniques for research on archaeozoological collections from the Middle East". En Becker, C., Manhart, H., Peters, C. y Schibler, J. (eds), Historia Animalium ex Ossibus. Beiträge zur Paläoanatomie, Archäologie, Ägyptologie, Ethnologie und Geschichte der Tiermedizin. Festschrift für Angela von den Driesch zum 65. Geburtstag. Internationale Archäologie, Studia honoraria 8. Rahden/Westfalen: 285-300.

Méndez, C. y D. Jackson 2008. "La ocupación prehispánica de Combarbalá (Norte Semiárido, Chile): una propuesta sintética”. Chungará 40(2):107-1 I9.

Rivera, B., P. López, I. Cartajena, B. Santander y C. Opazo. 2012. "Caracterización de las ocupaciones de los Periodos Intermedio Tardio y Tardio ( I.000-I.536 anos D.C.) en el Valle de Mauro (IV Región, Chile) a partir del registro zooarqueológico". Trabajo presentado al II Encuentro Latinoamericano de Zooarqueología.

Troncoso, A. 1999. "La Cultura Diaguita en el valle de Illapel: Una perspectiva exploratoria”. Chungará 30(2): I25- I 42.

200I.'La cultura Diaguita en el 200I, problemas y perspectivas desde el Choapa". Actas del IV Congreso Chileno de Antropología, tomo II, pp. I35 I-1356. Colegio de Antropólogos de Chile, Santiago.

2004. "Relaciones socio-culturales de producción, formas de pensamiento y ser en el mundo: un acercamiento a los períodos Intermedio Tardío y Tardío en la cuenca del río Choapa". Revista Werken 5: 61-68.

Troncoso, A., Becker, C., Pavlovic, D., González, P., Rodríguez, J. y C. Solervicens 2009. "El sitio LV099-B Fundo Agua Amarilla y la ocupación del período incaico en la costa de la provincia del Choapa”. Chungará 4 I (2): 24I-259. 